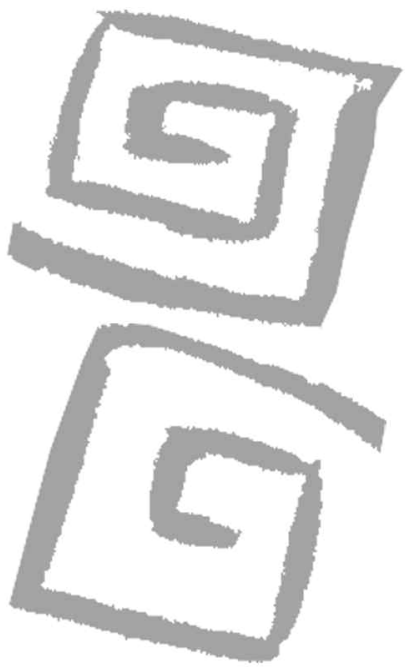

\title{
Validez y confiabilidad del Índice de Capacidad para el Trabajo en trabajadores del primer nivel de atención de salud en Argentina
}

\author{
Validity and reliability of the Work Ability Index \\ in primary care workers in Argentina
}

Peralta, Norma ${ }^{1}$; Godoi Vasconcelos, Ana Glória²; Härter Griep, Rosane33; Miller, Leticia ${ }^{4}$

\footnotetext{
${ }^{1}$ Licenciada en Enfermería. Enfermera del Departamento de Vigilancia y Clínica Epidemiológica. Especialista en Docencia Universitaria. Instituto Nacional de Epidemiologia "Dr. Juan H. Jara", Argentina. mnorma_peraltay@ahoo.com.ar

${ }^{2}$ Doctora en Ingeniería Biomédica. Investigadora adjunta, Escola Nacional de Saúde Pública. Coordinadora Adjunta del Programa de Epidemiología en Salud Pública. Fundação Oswaldo Cruz, Escola Nacional de Saúde Pública, Brasil. anagodoi@ensp.fiocruz.br

${ }^{3}$ Doctora en Ciencias de la Salud. Investigadora en Salud Pública del Laboratório de Educação em Ambiente e Saúde, Instituto Oswaldo Cruz, Fundação Oswaldo Cruz, Brasil. rohgriep@ioc.fiocruz.br

${ }^{4}$ Médica. Jefa del Departamento de Enseñanza Especializada, Instituto Nacional de Epidemiología "Dr. Juan H. Jara", Argentina. Imiller@ine.gov.ar
}

RESUMEN Este estudio evalúa la validez y confiabilidad del Índice de Capacidad para el Trabajo (ICT) en Argentina. El instrumento se aplicó a 100 trabajadores, agentes de Atención Primaria de la Salud del Partido de General Pueyrredón. En la validez del constructo se investigó la estructura dimensional por medio de análisis factorial exploratorio, a partir de una matriz policórica y un análisis paralelo para la obtención del número de factores. En la validación correlacional se estimó la correlación de Spearman entre el ICT y las dimensiones del Cuestionario de Salud SF-36. La evaluación de confiabilidad se midió a través del estimador Alfa de Cronbach. El resultado de la consistencia interna de la escala fue 0,80 , indicando una fiabilidad aceptable. La puntuación de ICT arrojó como resultado: moderado $12 \%$, bueno $50 \%$ y óptimo $38 \%$. En el proceso de validación se identificó una estructura de tres dimensiones que explica el $66 \%$ de la variación total de los datos a través de los componentes principales. Los supuestos teóricos de la validez del constructo confirmaron la correlación directa y significativa con las puntuaciones del ICT y las dimensiones de evaluación del estado de salud, siendo el mayor valor función física $(0,478)$ y menor la dimensión dolor $(-0,218)$. Se concluyó que el ICT, traducido y adaptado al español, mostró propiedades psicométricas adecuadas pudiendo ser utilizada en estudios de asociación entre aspectos de trabajo y su impacto en la salud.

PALABRAS CLAVE Personal de Salud; Validez de las Pruebas; Argentina.

ABSTRACT This study evaluates the validity and reliability of the Work Ability Index (WAI) in Argentina. An instrument was applied to 100 workers, all agents of Primary Health Care in the county of General Pueyrredón. In the construct validity, the dimensional structure was studied by means of exploratory factor analysis, based on a polychoric matrix and parallel analysis to obtain the number of factors. In the correlation validation, the Spearman correlation was estimated between the WAI and the dimensions of the 36Item Short Form Health Survey (SF-36). The reliability assessment was measured by Cronbach's alpha estimate. The result of the internal consistency of the scale was 0.80 , indicating acceptable reliability. The WAI score yielded the following results: $12 \%$ moderate, $50 \%$ good and $38 \%$ optimal. In the validation process, a three-dimensional structure was identified which accounts for $66 \%$ of the total variance of the data through the main components. The theoretical assumptions of the construct validity confirmed the direct and significant correlation between WAI scores and the dimensions of health status assessment, with the highest value in the physical functioning dimension (0.478) and the lowest in the bodily pain dimension (-0.218). It was concluded that the WAI, translated and adapted into Spanish, showed adequate psychometric properties and can therefore be used in association studies between aspects of work and their impact on health. KEY WORDS Health Personnel; Validity of Tests; Argentina. 


\section{INTRODUCCIÓN}

La dimensión que ha adquirido el concepto de salud en los últimos años, incorpora la influencia de las condiciones de trabajo y la percepción de incomodidad e insatisfacción del propio individuo como componentes fundamentales del concepto de calidad de vida. Este cambio de perspectiva interesa al área de gestión de personal, para la cual uno de los más importantes desafíos es saber si existen factores influyentes que puedan estimular o impedir el desarrollo de la tarea de las personas a lo largo de su vida laboral y condicionar su salud en el futuro (1-7).

El concepto de "capacidad para el trabajo" fue concebido con la finalidad de conocer al que genera recursos que, asociado a la medición de demandas físicas, mentales, estructura organizacional y ambiente de trabajo, permiten estimar indicadores específicos en el área laboral. Este concepto expresa:

...cómo es o será de capaz un/a trabajador/a en el presente o en el futuro, y cómo él o ella puede realizar su trabajo de acuerdo con los requisitos de su estado de salud y capacidades físicas y mentales. (8)

La implementación del Índice de Capacidad para el Trabajo (ICT) se fundamenta en la salud de los trabajadores, el proceso de envejecimiento de la población activa, el mantenimiento del trabajador productivo y la calidad de vida al momento de su jubilación (9).

Finlandia ostenta la condición de pionera en la investigación acerca del ICT, el cual surgió para evaluar el rendimiento actual de los trabajadores y estimar las condiciones de salud individuales con el objetivo de mejorarla, mirando si su capacidad futura se corresponde con las demandas de trabajo (9). De acuerdo con Ilmarinen y Tuomi $(8,10)$ el ICT ha permitido avanzar en la adaptación de las capacidades individuales a las necesidades del puesto de trabajo, lo cual redunda en la disminución del estrés y en la optimización de la eficiencia del trabajador teniendo, en consecuencia, mayores chances de preservar su salud física y psíquica y una mejor calidad de vida (9).
Desde 1981, el uso del ICT, tanto en la investigación como en la práctica, se ha empleado en varios países y se ha traducido a más de 24 idiomas, entre los cuales se encuentra la versión en español elaborada en Cuba (10).

No fue posible identificar investigaciones sobre las propiedades psicométricas del ICT en Argentina, lo que es fundamental para usarlo en estudios de asociación entre el índice y eventos de salud en el país. En este estudio se evalúan aspectos de la validez y confiabilidad del ICT en español en la Argentina.

\section{MÉTODOS}

\section{Población de estudio}

Se realizó un estudio epidemiológico transversal durante el mes de julio del año 2010. El universo poblacional estuvo compuesto por 152 profesionales (censo), que desempeñan actividades en Atención Primaria de la Salud en los centros de salud del Sistema Sanitario Municipal del Partido de General Pueyrredón que atienden las 24 horas. Participaron efectivamente 100 personas y las pérdidas se relacionaron con: negativas a participar de la investigación ( $n=29,19,08 \%$ ), licencia médica $(n=12,7,89 \%)$ y no localizados $(n=11,7,23 \%)$. Del total de trabajadores que aceptó responder la encuesta, el $72 \%$ era de sexo femenino, el $59 \%$ casado o en pareja, $31 \%$ soltero, $8 \%$ separado y $2 \%$ viudo. Con relación al tipo de profesión, 35\% eran médicos, 26\% enfermeros y $39 \%$ desarrollaban otras profesiones vinculadas con el primer nivel de atención (psicólogo, odontólogo, farmacéutico, asistente social, etc.).

La media de edad de la población estudiada fue de 39,01 años, con un rango de 24 a 64 años; presentaron una media de 8,61 años de trabajo en el municipio (desvío poblacional=8,89 años- y 4,75 años de continuidad de trabajo en el mismo centro (desvío poblacional $=5,58$ años). La media de antigüedad laboral del personal contratado fue de 1,57 años $(n=20$, desvío poblacional $=1,24$ años), y para la planta permanente de 7,62 años ( $n=80$, desvío poblacional $=7,72$ años).

El $67 \%$ tenía otra actividad laboral, la que en un $45 \%$ era realizada en otra institución no perteneciente al ámbito municipal. 


\section{Revisión de la traducción del instrumento Índice de Capacidad para el Trabajo}

A los fines de evaluar la equivalencia semántica de la traducción de la encuesta ICT versión inglés al español generada en Cuba, se convocaron tres expertos, cuyo perfil estuvo delimitado por el manejo acreditado de los dos idiomas en el campo de la salud. El objetivo principal fue poner a prueba las diez preguntas que constituyen la escala desde el punto de vista del significado de los términos utilizados en la traducción.

Para su validación, se utilizaron como referencia dos criterios:

1. En términos generales, si existió alteración en la traducción (alterada/no alterada).

2. En términos literales, si se produjo cambio de sentido.

La validación se realizó en escala de Likert: 5 totalmente de acuerdo, 4 de acuerdo, 3 neutral (afirmación), 2 en desacuerdo, 1 totalmente en desacuerdo.

\section{Instrumento para medir el ICT}

El cuestionario ICT está compuesto por siete dimensiones y diez preguntas de las cuales se obtienen las puntuaciones (11) (Cuadro 1). El resultado final oscila entre 7 y 49 puntos. El cálculo de la puntuación global tiene en cuenta la suma de los puntajes obtenidos en cada pregunta de acuerdo al Cuadro 1 (12). Al final, se clasifica al sujeto en cuanto a su capacidad para trabajar como: de capacidad pobre (7-27), moderada (28-36), buena (37-43) u óptima (44-49).

\section{Capacitación de los entrevistadores}

Dentro del proceso de investigación se administró el cuestionario estructurado, codificado con anterioridad. Se llevó a cabo de dos maneras, se elaboró material escrito bibliográfico (en el cual se explicitó el tipo de terminología que contiene la encuesta), se seleccionó a tres encuestadores de un grupo de estudiantes avanzados de la Tecnicatura de Seguridad e Higiene en el Trabajo de una universidad privada, cuya formación se relaciona con la salud y la seguridad de los trabajadores. La capacitación tuvo como finalidad:

- Preparar y sensibilizar al equipo de trabajo para la realización de la encuesta.

- Brindar al equipo de trabajo los conocimientos y la capacitación técnica necesaria pare Ilevar a cabo tal metodología.

- Brindar conocimiento acerca de la población de estudio, su localización laboral y distribución geográfica dentro del Partido de General Pueyrredón.

- Brindar información del material y los contenidos de cada ítem de la encuesta.

Estas finalidades son importantes, ya que la actitud y el comportamiento del equipo de trabajo podrían influir tanto en la calidad de la información como en la capacidad del grupo para obtener cuantitativamente respuesta poblacional.

\section{Pretest del instrumento}

Fue realizado con 15 personas, que posteriormente no fueron incluidas en el estudio. Se desarrolló encuestando al personal de un organismo nacional de salud que cumple funciones similares a las de la población objetivo del estudio.

Cuadro 1. Dimensiones utilizadas para medir el Índice de Capacidad para el Trabajo.

\begin{tabular}{lc}
\hline Dimensiones & Total \\
\hline $\begin{array}{l}\text { 1. Estimación subjetiva de la capacidad para el trabajo } \\
\text { actual comparada con la mejor de toda la vida }\end{array}$ & $0-10$ \\
$\begin{array}{l}\text { 2. Estimación subjetiva de la capacidad para el trabajo } \\
\text { relacionada con las demandas de trabajo (físicas y } \\
\text { mentales) }\end{array}$ & $2-10$ \\
$\begin{array}{l}\text { 3. Número de enfermedades actuales diagnosticadas } \\
\begin{array}{l}\text { 4. Estimación subjetiva de las limitaciones laborales } \\
\text { debidas a enfermedades }\end{array}\end{array}$ & $1-7$ \\
$\begin{array}{l}\text { 5. Ausentismo por enfermedad durante el último año } \\
\begin{array}{l}\text { 6. Pronóstico propio sobre la capacidad para los } \\
\text { próximos dos años }\end{array}\end{array}$ & $1-6$ \\
$\begin{array}{l}\text { 7. Recursos mentales (disfrute de las tareas diarias, } \\
\text { optimismo sobre el futuro) }\end{array}$ & $1-7$ \\
$\begin{array}{l}\text { Rango } \\
\text { Rano }\end{array}$ & $1-4$
\end{tabular}

Fuente: Tuomi (12) 
El proceso de ensayo en la aplicación del test tuvo las siguientes finalidades:

- Determinar el tiempo que insume la entrevista.

- Determinar si las preguntas eran lo suficientemente claras para la interpretación de los encuestados (información obtenida a través de un encuestador).

\section{ANÁLISIS DE DATOS}

\section{Validez del constructo del ICT}

La validez del constructo (a), establecida a partir de un conjunto de técnicas que buscan evaluar el grado en que una medida empírica refleja adecuadamente el significado real del concepto considerado (13), fue evaluada por medio de la validación correlacional y la validación factorial.

A los efectos de la validación correlacional, las personas respondieron a la encuesta del ICT y también al Cuestionario de Salud SF-36 (14), considerado un método psicométricamente sólido para medir la salud desde el punto de vista de la persona, ampliamente utilizado en el mundo y validado en Argentina, cuyo objetivo fue comparar la percepción del estado de salud, comparados a través de análisis factorial. La validación correlacional fue estimada a través de la correlación de Spearman (con niveles de significancia del 5\%) para verificar la concordancia entre el ICT y las dimensiones de la escala SF-36.

La estructura dimensional o validación factorial forma parte de la validación del constructo investigada por medio de análisis factorial exploratorio, que es una técnica estadística desarrollada sobre un supuesto de que una serie de variables observadas o medidas, Ilamadas variables empíricas u observacionales, pueden ser explicadas por un número menor de variables hipotéticas no observables Ilamadas factores (15). La factorabilidad de los datos se analizó mediante el índice de Kaiser-Meyer-Olkin (KMO) (16), que indica la proporción de varianza de los datos común a todas las variables, considerado muy útil cuando el tamaño muestral es pequeño. Valores bajos del índice KMO desaconsejan la utilización del ICT. Los criterios adoptados para la validación del test fueron: 1 a 0,9 muy bueno;
0,9 a 0,8 bueno; 0,8 a 0,7 medio; 0,7 a 0,6 razonable; 0,6 a 0,5 débil; <0,5 inaceptable.

Para identificar el número de factores que debe conservarse se utilizó análisis paralelo (b) (1). Luego se procedió a realizar un análisis factorial exploratorio, recurriendo al método de componentes principales. Se estipuló una carga de factor superior a 0,40. Para la rotación de la matriz de las cargas factoriales se utilizaron los procesos ortogonales con rotación varimax.

Mientras que la mayoría de las variables que componen el ICT son ordinales categóricas, a excepción de la variable "capacidad para el trabajo actual en comparación con la mejor de toda la vida" que es continua, se utilizaron para el análisis factorial correlaciones policóricas (c) (entre las variables ordinales) y correlación punto biserial (d) (variables continuas y ordinales).

La confiabilidad en este estudio se midió a través de la consistencia interna (e) mediante el índice Alfa de Cronbach para la puntuación global y también excluyendo ítem por ítem (18).

\section{Aspectos éticos}

El trabajo fue aprobado por el Comité de Bioética del Instituto Nacional de Epidemiología "Dr. Juan H. Jara" y el Comité de Ética del Municipio del Partido de General Pueyrredón, previa conformidad para adherir al proyecto. Cada trabajador firmó el consentimiento previo a la entrevista.

\section{RESULTADOS}

\section{Revisión de la traducción del instrumento Índice de Capacidad para el Trabajo}

Los expertos acordaron clasificar la comparación de las preguntas traducidas del inglés a la versión cubana en tres categorías: ítems equivalentes (manejo de propiedades sintácticas y léxico igual al original), ítems con modificaciones menores (vocabulario no igual pero que no modificara el sentido de la pregunta), ítems no equivalentes (la versión ofrecía distinta redacción e interpretación). Solo se 
observaron diferencias en dos preguntas con modificaciones menores en el vocabulario que no afectaron su sentido.

En el Cuadro 2, se observa que la población de estudio presentó elevados valores en las diversas dimensiones del ICT. La mayoría de los trabajadores ocupó las tres puntuaciones superiores de las dimensiones de "capacidad para el trabajo comparada con la mejor de toda la vida" (75\%); también en la dimensión de capacidad para el trabajo con relación a las exigencias del trabajo (79\%). Cerca del $44 \%$ refirieron no tener enfermedades actualmente diagnosticadas por un profesional médico, 3\% refirieron cinco o más enfermedades, 38\% refirieron no tener ninguna enfermedad que impida la realización de su trabajo y $4 \%$ considera ser capaz de hacer su trabajo, manifestando algún síntoma. Gran parte (66\%) consignó ausencia en el trabajo debido a enfermedad y el 96\% ocupó la puntuación más elevada en términos de apreciar su capacidad para el trabajo de aquí a dos años, y por último el $63 \%$ de los trabajadores ocupó la puntuación más elevada en términos de recursos mentales (capacidad para apreciar las actividades diarias, percepción de estar activo y alerta, sentimiento de esperanza para el futuro).
No hubo registro de puntuación de ICT bajo (7-27 puntos); 12\% registró una puntuación moderada (28-36), 50\% buena (37-43) y $38 \%$ óptima (44-49).

Los resultados del test de Kaiser-MeyerOlkin $(\mathrm{KMO}=0,70)$, muestran una adecuación de los datos regular para análisis factorial (19).

El análisis paralelo por componentes principales indicó 3 factores para ser extraídos. Esta indicación coincide con el número de autovalores mayores que 1 , otro criterio comúnmente utilizado con el mismo objetivo.

En el Cuadro 3, los tres factores extraídos explican el $66 \%$ de la varianza total de los datos (f). Si se considera el punto de corte 0,50 para el factor I, se advierte que reúne los ítems "Capacidad para el trabajo comparada con la mejor de toda la vida", "Capacidad para el trabajo con relación a las exigencias físicas del trabajo", "Capacidad para el trabajo con relación a las exigencias mentales del trabajo", "Sentimiento de estar activo y alerta" y "Esperanza para el futuro", con un valor propio de 3,46 explicando el $31 \%$ de la varianza total. Para el factor II, compuesto por "Ausencias en el trabajo por enfermedad en los últimos doce meses" y "Pronóstico personal sobre capacidad para el trabajo de aquí

Cuadro 2. Distribución de las respuestas de acuerdo a la puntuación en las diferentes dimensiones del Índice de Capacidad para el Trabajo, versión en español. Municipio del Partido de General Pueyrredón, Mar del Plata, 2010.

\begin{tabular}{|c|c|c|c|c|c|c|c|c|c|c|c|c|}
\hline \multirow[t]{2}{*}{ Dimensiones } & \multicolumn{12}{|c|}{ Puntuaciones } \\
\hline & 0 & 1 & 2 & 3 & 4 & 5 & 6 & 7 & 8 & 9 & 10 & Total \\
\hline $\begin{array}{l}\text { 1. Capacidad para el trabajo } \\
\text { comparada con la mejor de } \\
\text { toda la vida }\end{array}$ & 0 & 0 & 0 & 1 & 1 & 3 & 4 & 16 & 39 & 15 & 21 & 100 \\
\hline $\begin{array}{l}\text { 2. Capacidad para el trabajo con } \\
\text { relación a las exigencias laborales }\end{array}$ & - & - & 0 & 0 & 0 & 3 & 6 & 12 & 35 & 23 & 21 & 100 \\
\hline $\begin{array}{l}\text { 3. Número actual de enfermedades } \\
\text { diagnosticadas por un médico }\end{array}$ & - & 3 & 4 & 7 & 17 & 25 & - & 44 & - & - & - & 100 \\
\hline $\begin{array}{l}\text { 4. Pérdida de trabajo estimada } \\
\text { debido a enfermedades }\end{array}$ & - & 0 & 0 & 3 & 18 & 41 & 38 & - & - & - & - & 100 \\
\hline $\begin{array}{l}\text { 5. Ausencias en el trabajo por } \\
\text { enfermedad en los últimos doce } \\
\text { meses }\end{array}$ & - & 0 & 3 & 5 & 26 & 66 & - & - & - & - & - & 100 \\
\hline $\begin{array}{l}\text { 6. Pronóstico personal sobre capa- } \\
\text { cidad para el trabajo de aquí a } \\
\text { dos años }\end{array}$ & - & 0 & - & - & 4 & - & - & 96 & - & - & - & 100 \\
\hline 7. Recursos mentales & - & 0 & 8 & 29 & 63 & - & - & - & - & - & - & 100 \\
\hline
\end{tabular}

Fuente: Elaboración propia. 
a dos años" con un valor propio de 1,72 explicó el $18 \%$ de la varianza total. Para el factor III, "Número actual de enfermedades diagnosticadas por un médico" y "Pérdida de trabajo estimada debido a enfermedades" con un valor propio de 1,23 explicó el $17 \%$ de la varianza total. El ítem "Apreciación de actividades diarias" posee cargas factoriales semejantes en los factores I $(0,55)$ y II $(0,50)$.

El Cuadro 4 expresa la confiabilidad del Índice de Capacidad para el Trabajo medida por el coeficiente Alfa de Cronbach; su valor total fue de 0,80 y la exclusión de cualquiera de las preguntas no alteró sustancialmente el resultado.
En lo que se refiere a la validación del constructo del aspecto correlacional, el ICT presentó una correlación estadísticamente significativa $(p<0,01)$ con todas las dimensiones del estado de salud del Cuestionario de Salud SF-36. Considerando que a mejor estado de salud corresponde mayor valor del ICT, las correlaciones se presentaron en el sentido esperado, siendo las mayores correlaciones con función física (FFI) 0,478, rol emocional (Rem) 0,43 y salud mental (SM) 0,41. Presentó una correlación inversa con el dolor, -0,22, lo que demuestra que a menor dolor mayor ICT (Cuadro 5).

Cuadro 3. Análisis de los tres componentes principales del Índice de Capacidad para el Trabajo, con rotación varimax $(n=100)$.

\begin{tabular}{|c|c|c|c|c|c|}
\hline \multirow[t]{2}{*}{ Dimensiones } & \multirow[t]{2}{*}{ Preguntas } & \multicolumn{4}{|c|}{ Componentes principales } \\
\hline & & Factor I & Factor II & Factor III & Comunalidad \\
\hline 1 & $\begin{array}{l}\text { Pl. Suponga que la mejor capacidad } \\
\text { para el trabajo que ha tenido en su vida } \\
\text { recibe un valor de } 10 \text { puntos. ¿Cuántos } \\
\text { puntos daría a la capacidad para el tra- } \\
\text { bajo que tiene actualmente? }\end{array}$ & 0,64 & 0,25 & 0,10 & 0,48 \\
\hline \multirow[t]{2}{*}{2} & $\begin{array}{l}\text { P2a. ¿ Cómo califica su capacidad para } \\
\text { el trabajo actual con respecto a las } \\
\text { demandas físicas de su actividad laboral? }\end{array}$ & 0,78 & $-0,08$ & 0,18 & 0,64 \\
\hline & $\begin{array}{l}\text { P2b. ¿Cómo califica su capacidad para } \\
\text { el trabajo actual con respecto a las } \\
\text { demandas mentales de su trabajo? }\end{array}$ & 0,80 & $-0,09$ & 0,16 & 0,67 \\
\hline 3 & $\begin{array}{l}\text { P3. En la siguiente lista, marque las } \\
\text { enfermedades o trastornos que pade- } \\
\text { ce. Por cada enfermedad de la lista } \\
\text { puede indicar: en su opinión, si estas } \\
\text { han sido diagnosticadas o tratadas por } \\
\text { un médico, o bien no marcar ninguna } \\
\text { de estas alternativas. }\end{array}$ & 0,12 & 0,14 & 0,92 & 0,88 \\
\hline 4 & $\begin{array}{l}\text { P4. } \dot{2} \text { Es su enfermedad o padecimiento } \\
\text { un impedimento para su trabajo? }\end{array}$ & 0,27 & 0,40 & 0,81 & 0,88 \\
\hline 5 & $\begin{array}{l}\text { P5. ¿Cuántos días completos ha tenido } \\
\text { que dejar de trabajar debido a proble- } \\
\text { mas de salud (enfermedad, o realiza- } \\
\text { ción de exámenes médicos) durante el } \\
\text { último año ( } 12 \text { meses)? Ausencias en el } \\
\text { trabajo por enfermedad en los últimos } \\
\text { doce meses. }\end{array}$ & $-0,06$ & 0,63 & 0,37 & 0,53 \\
\hline 6 & $\begin{array}{l}\text { P6. ¿Ud. cree, de acuerdo con su salud, } \\
\text { que será capaz de hacer su trabajo } \\
\text { actual de aquí a dos años? }\end{array}$ & 0,21 & 0,92 & $-0,15$ & 0,91 \\
\hline \multirow[t]{5}{*}{7} & $\begin{array}{l}\text { P7a. ¿Últimamente se ha sentido capaz } \\
\text { de disfrutar sus actividades cotidianas? }\end{array}$ & 0,55 & 0,50 & 0,02 & 0,56 \\
\hline & $\begin{array}{l}\text { P7b. ¿Últimamente se ha sentido activo y } \\
\text { dispuesto? }\end{array}$ & 0,75 & 0,23 & $-0,02$ & 0,62 \\
\hline & $\begin{array}{l}\text { P7c. ¿Últimamente se ha sentido espe- } \\
\text { ranzado y animado para el futuro? }\end{array}$ & 0,64 & 0,15 & 0,09 & 0,54 \\
\hline & Varianza de componente & 0,31 & 0,18 & 0,17 & \\
\hline & Varianza de componente & 0,31 & 0,49 & 0,66 & \\
\hline
\end{tabular}


Cuadro 4. Valor de Alfa de Cronbach si el ítem es excluido y para la puntuación global del Índice de Capacidad para el Trabajo en trabajadores del primer nivel de atención de salud. Municipio del Partido de General Pueyrredón, Mar del Plata, 2010 (n =100).

\begin{tabular}{|c|c|}
\hline Ítems & $\begin{array}{l}\text { Alfa de } \\
\text { Cronbach } \\
\text { según pregunta } \\
\text { excluida }\end{array}$ \\
\hline $\begin{array}{l}\text { P1. Capacidad para el trabajo comparada } \\
\text { con la mejor de toda la vida }\end{array}$ & 0,78 \\
\hline $\begin{array}{l}\text { P2a. Capacidad para el trabajo con relación } \\
\text { a las exigencias físicas del trabajo }\end{array}$ & 0,78 \\
\hline $\begin{array}{l}\text { P2b. Capacidad para el trabajo con relación } \\
\text { a las exigencias mentales del trabajo }\end{array}$ & 0,78 \\
\hline $\begin{array}{l}\text { P3. Número actual de enfermedades } \\
\text { diagnosticadas por un médico }\end{array}$ & 0,81 \\
\hline $\begin{array}{l}\text { P4. Pérdida de trabajo estimada debido a } \\
\text { enfermedades }\end{array}$ & 0,77 \\
\hline $\begin{array}{l}\text { P5. Ausencias en el trabajo por enfermedad } \\
\text { en los últimos doce meses }\end{array}$ & 0,81 \\
\hline $\begin{array}{l}\text { P6. Pronóstico personal sobre capacidad } \\
\text { para el trabajo de aquí a dos años }\end{array}$ & 0,79 \\
\hline P7a. Apreciación de las actividades diarias & 0,78 \\
\hline P7b. Sentimiento de estar activo y alerta & 0,77 \\
\hline P7c. Esperanza para el futuro & 0,78 \\
\hline Total & 0,80 \\
\hline
\end{tabular}

Fuente: Elaboración propia.

\section{DISCUSIÓN}

Teniendo en cuenta el contexto actual de cambios en la producción, las relaciones laborales y el envejecimiento de la población activa en donde las cuestiones de la capacidad de los trabajadores de la salud y el trabajo cobran especial importancia, esta investigación sienta un precedente en términos de línea de base para futuras aplicaciones en la región y futuros análisis de evolución del ICT medido.

Por haberse aplicado la encuesta traducida en Cuba se utilizó una metodología estándar de corroboración de la traducción de la versión en inglés al español, que incluyó el análisis de expertos comprobando equivalencias semánticas, equivalencias de significado de las palabras y equivalencias utilizadas en las expresiones (20).

Se encontró en la población estudiada mayor porcentaje del ICT bueno y moderado, con un $38 \%$ de óptimo.

Estudios epidemiológicos han investigado la asociación de la edad con la pérdida temprana de la capacidad para el trabajo (21) y se observó que esta variable no muestra diferencias respecto de la pérdida de capacidad laboral, lo que corrobora los resultados obtenidos en esta investigación.

Los estudios sobre la estructura dimensional del ICT han Ilegado a resultados diferentes. Este estudio identificó por medio de componentes principales que los ítems se agregan en tres dimensiones, explicando el $66 \%$ de la varianza total. En Brasil, un estudio realizado por Martínez et al. (22) con trabajadores de electricidad, identificó a través de componentes principales, tres factores con valores propios superiores a 1, explicando el $57,9 \%$ de la varianza. Almeida da Silva Junior (23) utilizando también componentes principales y rotación de varimax, encontró dos factores explicando el $53 \%$ de la varianza de los datos en profesionales de enfermería.

En términos de estructura factorial, el primer factor identificado en este estudio agrega los ítems en forma similar a lo encontrado en el estudio de Almeida da Silva Junior (23), ambos con profesionales del área de salud.

En el estudio de Martínez et al. (22), el primer factor con el $20,6 \%$ de la varianza fue representado por los elementos "Apreciación de las actividades diarias", "Sentimiento de estar activo y alerta" y "Esperanza para el futuro", que representa la dimensión "Recursos mentales". El segundo factor con el $18,9 \%$ de la varianza se compone de los elementos "Capacidad para el

Cuadro 5. Correlación de Spearman del Índice de Capacidad para el Trabajo global con dimensiones del Cuestionario de Salud SF-36.

\begin{tabular}{lcc}
\hline $\begin{array}{l}\text { Dimensiones del } \\
\text { Cuestionario de Salud SF-36 }\end{array}$ & $r$ & p-valor \\
\hline Función física & 0,478 & $<0,01$ \\
Rol físico & 0,316 & $<0,01$ \\
Dolor & $-0,218$ & $<0,05$ \\
Salud & 0,287 & $<0,01$ \\
Vitalidad & 0,323 & $<0,01$ \\
Función social & 0,364 & $<0,01$ \\
Rol emocional & 0,423 & $<0,01$ \\
Salud mental & 0,421 & $<0,01$
\end{tabular}

Fuente: Elaboración propia. 
trabajo comparada con la mejor de toda la vida", "Capacidad de trabajo en relación con las exigencias físicas del trabajo" y "Capacidad para el trabajo en relación con las exigencias mentales del trabajo" que representa la percepción del trabajador en cuanto a su capacidad para trabajar. El tercer factor con el 18,4\% de la varianza se compone de los elementos "Número actual de enfermedades diagnosticadas por un médico", "Pérdida de trabajo estimada debido a enfermedades", "Ausencias del trabajo por enfermedad en los últimos 12 meses" y "Pronóstico personal sobre la capacidad para el trabajo de aquí a dos años" que están relacionados con la presencia de enfermedad y las restricciones en el marco del estado de salud.

La investigación de la validez correlacional mostró que las dimensiones del cuestionario SF-36 correlacionaron con el ICT con mayor valor igual a 0,48 $(p<0,01)$, mostrando una tendencia de mejores puntuaciones de capacidad para el trabajo que se correlacionan con mejor evaluación del estado de salud. Refiere también una correlación negativa con respecto al dolor $(-0,218)$.

Estos resultados permiten interpretar que el constructo "capacidad para el trabajo" comprendido como la capacidad que un trabajador tiene para ejercer sus tareas está determinado por las condiciones de salud física y mental (12).

Todas las dimensiones correspondientes al estado de salud correlacionaron estadísticamente con el ICT, mostrando que las dimensiones "función física", "rol emocional" y "salud mental" presentaron una correlación más fuerte. En general, la salud física presenta una correlación más sólida con el ICT que aquellas relacionadas con la salud mental (24). En este estudio, la mezcla de ambas dimensiones se justificaría con el tipo de actividad que realiza la población de estudio.

El tipo de diseño transversal de este estudio no infiere relaciones causales. Sin embargo, para explicar la alta calidad de la salud y la capacidad de trabajo de los trabajadores del primer nivel de atención, hay que considerar el efecto del trabajador sano, que se debe principalmente al proceso de selección para la admisión de los trabajadores y la exclusión de los que más frecuentemente se enferman o faltan al trabajo debido a enfermedades (25).

Este efecto es un fenómeno complejo y difícil de medir, pero se lo debe considerar al evaluar los resultados de estudios sobre salud del trabajador (25), a partir del ingreso a la actividad laboral. Aunque los datos de este estudio no permiten medir este efecto, no debe descartarse, ya que la utilización sistemática de este instrumento permitiría implementar medidas para preservar la capacidad laboral de los involucrados.

A pesar de los satisfactorios estándares del perfil de salud y la capacidad para el trabajo observadas en el presente estudio, un seguimiento del estado de salud que incluya el control médico periódico como rutina y la evaluación de la capacidad de trabajar a través del ICT y el SF36 para todos los trabajadores, permitiría la vigilancia rutinaria de los mismos, a nivel individual y colectivo, de manera tal que se identificarían los cambios tempranos, permitiendo aplicar medidas para mejorar las condiciones de trabajo.

Cabe también atender a la cuestión del estrés en el trabajo, puesto que aun cuando los niveles identificados no son altos, se sugiere la aplicación de medidas para mejorar la organización y el ambiente de trabajo psicosocial con el fin de satisfacer las necesidades y expectativas de los trabajadores acorde con las necesidades y características de la tarea.

Por otro lado habría que considerar otros factores relativos al ambiente de trabajo tratados en la literatura y no contemplados en el ICT -que abarca solo los aspectos relacionados con el trabajador- fundamentales para conocer las capacidades, limitaciones y necesidades de las personas, desconociendo los aspectos relacionados con el lugar y el ambiente de trabajo $(26,27)$.

Un objetivo indirecto de la validación del ICT fue demostrar su importancia para el mantenimiento de la capacidad para el trabajo, ya que el envejecimiento de la población plantea nuevos retos para la promoción de capacidad del trabajador. Por lo tanto, promover la capacidad para el trabajo puede aumentar el bienestar y la felicidad, así como la calidad del empleo.

Por tratarse de una población con características específicas en cuanto al perfil de salud (atención primaria de la salud) e inserción ocupacional, el resultado del ICT puede no ser válido para otras poblaciones de trabajadores del país, por lo que la confiabilidad del instrumento debería ser validada para cada población de estudio. 


\section{AGRADECIMIENTOS}

Este proyecto fue financiado por el Instituto Nacional de Epidemiología “Dr. Juan H. Jara" dependiente de la Administración Nacional de Laboratorios "Dr. Carlos Malbrán", Ministerio de Salud de la Nación.

\section{NOTAS FINALES}

a. Validez del constructo (rotación varimax): Debemos establecer estadísticamente grupos de ítems que deberán ser definidos como dimensiones. Estas dimensiones deben ser conceptualizadas de manera consensuada para otorgarles el carácter de útiles y científicamente válidas, por cuanto no existe una prueba patrón. Si los dominios se han construido teóricamente, la estadística corrobora la idoneidad de estas agrupaciones.

b. Análisis paralelo (26): El procedimiento está basado en el principio de que los factores a extraer deben dar cuenta de más varianza que la que es esperada de manera aleatoria. El procedimiento reordena las observaciones de manera aleatoria entre cada variable y los autovalores son recalculados a partir de esta nueva base de datos aleatoriamente ordenada. Los factores con autovalores mayores a los valores aleatorios son retenidos para la interpretación.

c. Correlación policórica: Es una estimación del grado de relación lineal entre dos variables continuas latentes a dos variables de naturaleza dicotómica u ordinal. Las correlaciones policóricas no asumen distribución normal multivariante de las variables observadas, y tampoco la asumen para las variables latentes.

d. Correlación punto biserial: Es el coeficiente de correlación que se aplica cuando se trata de cuantificar la relación que existe entre una variable dicotómica por esencia (por ejemplo, sexo), o dicotomizada por conveniencia, con otra variable cuantitativa. e. Consistencia interna (Alfa de Cronbach): Es la capacidad de obtener resultados independientes de las circunstancias accidentales de la medición. Se fundamenta en la varianza de la variable medida; existen varios índices que miden confiabilidad, entre ellos el Alfa de Cronbach y el Kuder-Richardson (KR-20). Es afectado por la heterogeneidad de los individuos evaluados: cuanto más heterogéneo es el grupo, mayor es el coeficiente de fiabilidad.

f. Comunalidad: Es la proporción de la varianza explicada por los factores comunes. Las comunalidades iniciales dentro del análisis de componentes principales son siempre iguales a uno, por lo que este dato no representa información importante. En cambio, en los estadísticos finales la comunalidad sí tiene una significación importante. Esto se debe a que al final del proceso no queda explicada la varianza total de cada una de las variables debido a que solo se ha conservado un pequeño conjunto de factores entre todos los posibles y, por lo tanto, la comunalidad de cada variable representará la proporción de varianza explicada por el conjunto de los factores finales resultantes. La comunalidad de cada variable se estima a partir de la matriz factorial y puede oscilar entre cero y uno. Cuando obtenemos una comunalidad de cero quiere decir que los factores comunes no tienen ningún poder explicativo de la variabilidad de una variable. En cambio, si la comunalidad es uno, entonces la variable está totalmente explicada por los factores comunes que aparecen en la matriz factorial. Por último, la varianza que no queda explicada por los factores comunes se atribuye al factor único, el cual no aparece en la matriz factorial.

\section{REFERENCIAS BIBLIOGRÁFICAS}

1. Karasek R, Theorell T. Health work, stress, productivity and reconstruction of working life. New York: Basic Books; 1991.

2. Gómez Vidal MA. Efectos tóxicos sobre la salud derivado de la exposición crónica a plaguicidas: Importancia de los marcadores bioquímicos. [Tesis de Doctorado] Granada: Facultad de Medicina, Universidad de Granada; 1999.
3. Hiba JC, director. Condiciones de trabajo, seguridad y salud ocupacional en la minería del Perú. Ginebra: Organización Internacional del Trabajo; 2002.

4. Mingote Adam JC. Satisfacción, estrés laboral y calidad de vida del médico. [Tesis de Doctorado]. Madrid: Universidad Autónoma de Madrid; 1994.

5. García Viniegras C, López Gonzalez V. Consideraciones teóricas sobre el bienestar y la 
salud. Revista Habanera de Ciencias Médicas. [Internet]. 2003-2004 [citado 10 mar 2012];2(7). Disponible en: http://bvs.sld.cu/revistas/rhab/articulo_rev7/carmen.html.

6. Robalino Campos M; Körner A. Condiciones de trabajo y salud docente: Estudios de casos en Argentina, Chile, Ecuador, México, Perú y Uruguay. Santiago de Chile: UNESCO; 2005.

7. Juárez García A. Factores psicosociales laborales relacionados con la tensión arterial y síntomas cardiovasculares en el personal de enfermería en México. Salud Pública de México. 2007;49(2):109117.

8. Ilmarinen J, Tuomi K. Past, present and future of work ability. En: Ilmarinen J, Lehtinen S, editors. Past, present and future of work ability. Helsinki: Finnish Institute of Occupational Health; 2004. p. 1-25. (People and Work, Research Reports No. 65).

9. Ilmarinen J, Tuomi K, Eskelinen L, Nygård CH, Huuhtanen $\mathrm{P}$, Klockars M. Background and objectives of the Finnish research project on aging workers in municipal occupations. Scandinavian Journal of Work, Environment \& Health. 1991;17(Suppl 1):S7-S11.

10. Tuomi K, Huuhtanen P, Nykyri E, Ilmarinen J. Promotion of work ability, the quality of work and retirement. Occupational Medicine. 2001;51(5):318-324.

11. Ilmarinen J, Tuomi K. Work ability of aging workers. Scandinavian Journal of Work, Environment \& Health. 1992;18(Suppl 2):S8-S10.

12. Tuomi K, Ilmarinen J, Jahkola A, Katajarinne L, Tulkki A. Índice de capacidade para o trabalho. Fischer FM, tradutor. São Carlos: EDUFSCar; 2005.

13. Griep RH, Chor D, Faerstein E, Werneck GL, Lopes CS. Validade de constructo de escala de apoio social do Medical Outcomes Study adaptada para o português no Estudo Pró-Saúde. Cadernos de Saúde Pública. 2005;21(3):703714.

14. Alonso J, Prieto L, Anto JM. La versión española del SF-36 Health Survey (Cuestionario de Salud SF-36): un instrumento para la medida de los resultados clínicos. Medicina Clínica. 1995; 104(20):771-776.

15. Pasquali L. Análise fatorial para pesquisadores. Brasília: LabPAM; 2005.
16. Mari JJ, Williams P. A validity study of a psychiatric screening questionnaire (SRQ-20) in primary care in the city of São Paulo. British Journal of Psychiatry. 1986;148:23-26.

17. Horn JL. A rationale and test for the number of factors in factor analysis. Psychometrika. 1965;30(2):179-185.

18. Revelle W. Psych: Procedures for Psychological, Psychometric, and personality research [Internet] 2012 [citado 10 may 2012]. Disponible en: http://www.personality-project. org/r/psych.manual.pdf.

19. Aaronson NK, Acquadro C, Alonso J, Apolone G, Bucquet D, Bullinger M, Bungay K, Fukuhara S, Gandek B, Keller S, et al. International quality of life assessment (Iqola) Project. Quality of Life Research. 1992;1(5):349-351.

20. Guillemin F, Bombardier C, Beaton D. Crosscultural adaptation of health-related quality of life measures: literature review and proposed guidelines. Journal of Clinical Epidemiology. 1993;46(12):1417-1432.

21. Ilmarinen J. Aging and work-coping with strengths and weaknesses. [Editorial]. Scandinavian Journal of Work, Environment \& Health. 1997;23 (Suppl 1):S3-S6.

22. Martinez MC, Latorre Dias de Oliveira MR. Validity and reliability of the Brazilian version of the Work Ability Index questionnaire. Revista de Saúde Pública [Internet]. 2009 [citado 4 jul 2011];43(3). Disponible en: http://www.scielo.br/ scielo.php?pid = S0034-89102009000300017 $\&$ script $=$ sci arttext\&tlng $=$ en .

23. Silva Junior SHA, Vasconcelos AGG, Griep RH, Rotenberg L. Validade e confiabilidade do índice de capacidade para o trabalho (ICT) em trabalhadores de enfermagem. Cadernos de Saúde Pública [Internet]. 2011 [citado 4 jul 2011];27(6):10771087. Disponible en: http://www.scielo.br/scielo. php? script $=$ sci arttext $\&$ pid $=$ S0102-311X2011 $000600005 \& \operatorname{lng}=$ pt.

24. Martinez MC, Latorre Dias de Oliveira MR. Saúde e capacidade para o trabalho de eletricitários do Estado de São Paulo. Ciência \& Saúde Coletiva. 2008;13(3):1061-1073.

25. Nygård CH, Eskelinen L, Suvanto S, Tuomi K, Ilmarinen J. Associations between functional capacity and work ability among elderly municipal employees. Scandinavian Journal of Work, Environment \& Health. 1991;17(Suppl 1):S122S127. 
26. García AM, Gadea R, Sevilla MJ, Genís S, Ronda E. Ergonomía participativa: emponderamiento de los trabajadores para la prevención de trastornos musculoesqueléticos. Revista Española de Salud Pública. 2009;83(4):509-518.
27. Catalano AM, Mendizábal N, Neffa JC. Condiciones y medio ambiente de trabajo y salud de los obreros de la industria del vidrio. Buenos Aires: Trabajo y Sociedad, PIETTE/CONICET, CREDAL/CNRS, SOIVA; 1993.

\section{FORMA DE CITAR}

Peralta N, Godoi Vasconcelos AG, Härter Griep R, Miller L. Validez y confiabilidad del Índice de Capacidad para el Trabajo en trabajadores del primer nivel de atención de salud en Argentina. Salud Colectiva. 2012;8(2):163-173.

Recibido el 2 de septiembre de 2011

Versión final presentada el 1 de febrero de 2012

Aprobado el 30 de marzo de 2012 